\title{
Interleaved Operation of Three-Level Neutral Point Clamped Converter Legs and Reduction of Circulating Currents under SHE-PWM
}

\begin{abstract}
Interleaving of voltage source converter legs enables higher output currents per phase, effectively increasing the power rating without increasing semiconductor ratings. However, the interleaved operation results in circulating currents between each phase converter legs as well as a zero-sequence circulating current which become quite prominent when the converters operate with low switching frequencies. This paper demonstrates the interleaved operation of three-level converters under selective harmonic elimination pulse-width modulation (SHE-PWM). A controller for the circulating current within the legs of each phase is also proposed. The controller is used in combination with optimally selected SHE-PWM patterns to generate the maximum number of voltage levels and minimise the peak value of the circulating current. Simulation and experimental results show the interleaved operation and the effect of SHE-PWM pattern selection in the overall system.
\end{abstract}

Index Terms-Interleaving, multilevel converters, multilevel waveforms, parallel legs, selective harmonic elimination, SHEPWM, voltage source converters,

\section{INTRODUCTION}

Interleaved parallel operation of voltage source converter (VSC) legs provides an effective way of increasing the power rating of a power electronics converter without the need for parallel connection of semiconductor devices [1][7] applicable to both two-level and multilevel converters [8][10]. Interleaved operation also increases the reliability and availability of supply [11] and is quite common in interruptible power supply (UPS) applications. The output of each converter leg that comprise one phase is combined with the use of inductors, generating a single output voltage. Assuming an ideal operation of the converters, the current should be equally shared amongst each of the converter legs, however this requirement cannot be readily satisfied unless actively controlled [5]. Furthermore, the finite switching frequency of the converter will result in circulating currents within the converter legs.

One of the main challenges associated with the interleaved connection of converter legs is the generation of a zerosequence circulating current (ZSCC) between the dc and ac side [1], [2]. The ZSCC leads to increased semiconductor rating requirements and further increases both conduction and switching losses of these topologies [12]. The mechanism of ZSCC is well understood and typical solutions include isolation of the ac and dc side, adding a high zero-sequence impedance or operating the parallel inverters with the same control [2]. The first of the three solutions adds to the overall complexity, while operating the two converters in the same way effectively removes their interleaving and reduces the quality of the combined output voltages and load currents.
Under relatively high switching frequencies (i.e. carrierbased pulse width modulation (CB-PWM) [13]-[15] or spacevector PWM (SV-PWM), control of the circulating current is achieved through adjustment of the reference for each converter leg, usually through PI controllers or exact reference generation [5], in order to compensate for any deviation. A method that calculates the power reference for each of the parallel converter legs with isolated supplies without input from the the other legs, applied to UPS, was proposed in [6], while a discontinuous modulation scheme, that allows the use of a single-core three-limb inductor, was presented in [7]. However, most of these methods consider high switching frequencies which might be prohibitive for high power applications, where switching losses and overall harmonic performance are major priorities.

Selective harmonic elimination pulse-width modulation (SHE-PWM) offers tight control of the low-order voltage harmonics and is well-suited for high-power, low switching frequency applications [16]. However, the timing of transitions is critical to the exact elimination of the harmonics; deviation from the pre-calculated patterns has an adverse effect on the performance of SHE-PWM. As a result, significant deviation from the pre-calculated patterns in order to control the circulating currents does not offer a viable solution.

Other solutions based on SHE-PWM focused on the minimisation of the ZSCC [12], [17]-[19] by introducing additional transitions and eliminating low-order triplen harmonics. Such methods effectively increase the converter switching frequency [12] and limit the range where SHE-PWM solutions can be found. Furthermore, inclusion of triplen harmonics in the SHEPWM formulation transforms the problem from three-phase to single-phase [20] while elimination of only certain triplen harmonics (e.g. 3rd or 9th harmonic) does not necessarily lead to lower ZSCC.

The objective of this paper is to demonstrate interleaving of three-level neutral-point clamped converter legs under SHEPWM and experimentally verify a controller for the circulating current between the converter legs. As the implementation does not aim to modify the SHE-PWM patterns, the main variables that drive the circulating current between a converter leg are also defined. The results are equally applicable to neutral-point clamped (NPC) and active NPC (ANPC) converters. The current article extends the preliminary work that was presented in [9] by providing the detailed evaluation of SHE-PWM patterns and a comprehensive set of experimental results for interleaved three-level converters.

This paper is organised in the following manner. Section II describes the concept of interleaved converters, circulating 


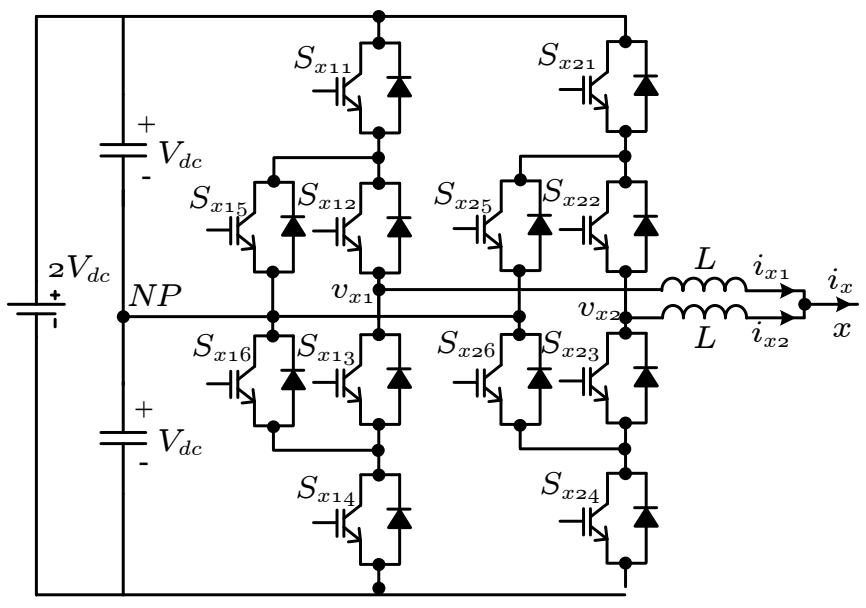

Fig. 1. One phase of the three-level ANPC converter with two interleaved converter legs.

current within the converter legs and its dependency on the switching states. Section III provides an overview of SHEPWM for interleaved multilevel converters, the effect of switching patterns on circulating currents and a comprehensive evaluation of the solutions. The controller for the circulating current is briefly described in Section IV and its performance is validated by simulation results, demonstrating three-phase system operation in Section V and experimental results from a single-phase laboratory prototype in Section VI. Finally, the conclusions of the work are summarized in Section VII.

\section{Parallel-Connected Converter Legs}

\section{A. Converter Configuration}

The work presented in this paper focuses on two-leg interleaved, three-level NPC converters, as shown in Fig. 1 in an ANPC configuration, where two interleaved converter legs form each of the phases. As the converter legs share the same dc-link and in order to avoid short-circuits between the legs, inductors $(L)$ are added to the output of each phase. The main function of $L$ is to limit the circulating current within the legs when the output voltage level of the two converters is different.

Each of the converter legs generates three voltage levels in its output, $v_{x j}, j \in[1,2]$. Based on the Thevenin equivalent, the combined output voltage is a result of the averaging between the two voltage outputs $\left(v_{x 1}\right.$ and $v_{x 2}$ in Fig. 1) and the phase output voltage $v_{x}$, where $x$ denotes the phase $x \in\{a, b, c\}$, is given as:

$$
v_{x}=\frac{v_{x 1}+v_{x 2}}{2} .
$$

Because of the averaging effect, the voltage $v_{x}$ has additional voltage levels (in the case of two interleaved three-level converters the maximum number of levels is five) effectively improving the harmonic performance of the converter. This concept can be further extended to $k$ three-level converters in parallel, generating $2 k+1$ levels in the Thevenin equivalent output voltage given by the averaging of each converter output voltage as

$$
v_{x}=\frac{1}{k} \sum_{j=1}^{k} v_{x j},
$$

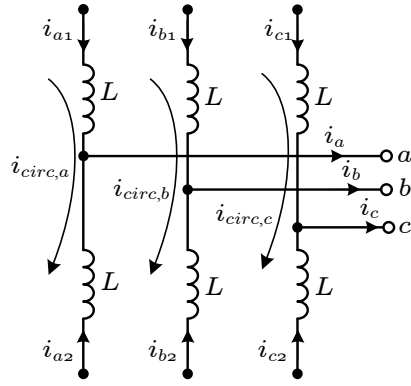

(a)

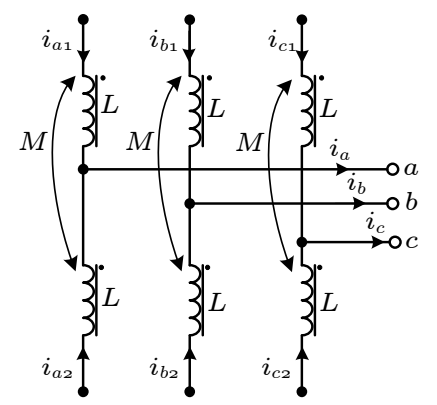

(b)
Fig. 2. (a) Single and (b) coupled inductors for interleaving of converter legs.

and $j \in[1, \ldots, k]$ denotes each of the $k$ interleaved converters. The proposed controller will make use of the maximum number of voltage levels from the converter in order to fully utilise the harmonic performance of the interleaved converters.

The use of a single inductor per leg (Fig. 2(a)) provides a more modular and expandable system compared to the coupled inductors, however, practical limits in the maximum impedance [10] and the non-optimal use of magnetic material in single inductors are significant drawbacks in practical applications. Coupled inductors (Fig. 2(b)) result in high differential mode impedance in the path between the interleaved converters, a concept that can be further extended to multiple interleaved converter either with one common core [4] or with multiple coupled inductors [5]. Ref. [21] investigated the ZSCC for interleaved converters with three-phase, three-pole transformers. In all previous cases, the effect of the circulating current is a function of the differential mode impedance and, in the following analysis, coupling between the inductors has not been considered.

\section{B. Converter Switching States and Circulating Currents}

Each state combination of the two interleaved converter legs (Fig. 3) has a different effect on the circulating current $\left(i_{\text {circ }, x}\right)$

$$
i_{\text {circ }, x}=\frac{i_{x 1}-i_{x 2}}{2}
$$

within one phase of the converter. The differential component of the voltage, $v_{x 1}-v_{x 2}$, is then imposed across both the inductors of the two converter legs. This effect can be further transformed to a weighted value through normalisation with the dc-link voltage $\left(V_{d c}\right)$ as also shown in Fig. 3.

Transitions between two different levels that involve simultaneous switching of both interleaved converters (shown in red arrows in Fig. 3) should be avoided as they lead to an unacceptable increase of the switching frequency. The top and bottom voltage levels, $+V_{d c}$ and $-V_{d c}$ respectively, are generated by a single combination which does not affect the circulating current. Additionally, the two zero-states that lead to excessive deviations in the circulating current $( \pm 2)$ are omitted (denoted with the red background in Fig. 3). The circulating current is affected only during the two redundant states of levels $+V_{d c} / 2$ and $-V_{d c} / 2$ (Fig. 3). These redundancies will be actively used to regulate $i_{c i r c, x}$. During the state combinations that generate $+V_{d c} / 2$ and $-V_{d c} / 2$, the 


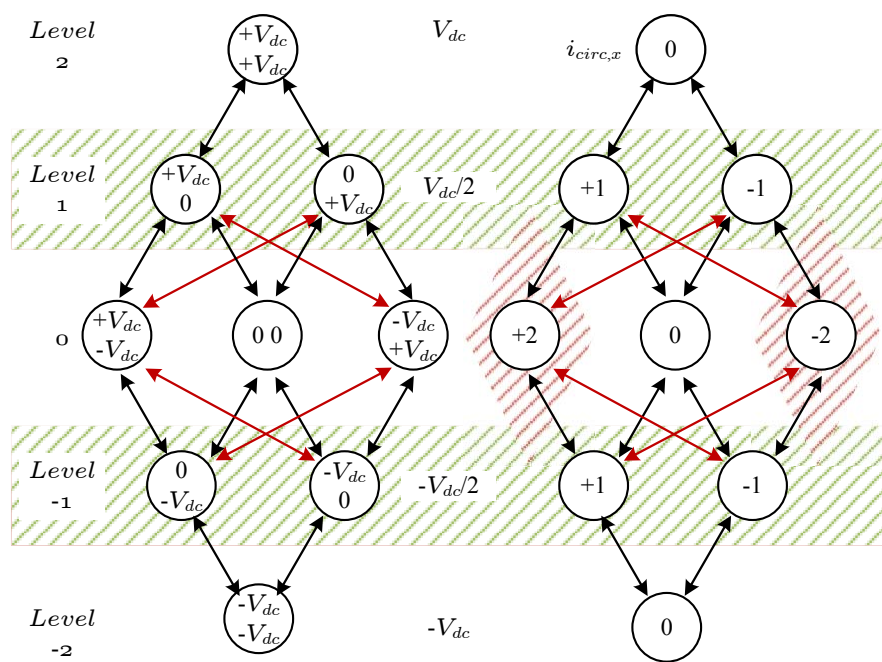

Fig. 3. Switching states of two three-level interleaved converters and their effect on the phase circulating current $i_{\operatorname{circ}, x}$

voltage applied across the two inductors is equal to $+V_{d c} / 2$ and $-V_{d c} / 2$ and the rate of change of the circulating current is defined as

$$
\frac{d i_{c i r c, x}}{d t}= \pm \frac{V_{d c}}{2 L}
$$

An additional component of interest in interleaved converter legs is that of the ZSCC [12], [18], [19], defined as

$$
i_{Z S C C}=i_{c i r c, a}+i_{c i r c, b}+i_{c i r c, c},
$$

which, together with individual circulating currents, should be kept within limits. However, minimising the ZSCC does not necessarily lead to a minimisation of the circulating currents within each phase.

\section{Multilevel SHE-PWM}

\section{A. Problem Formulation and Angle Calculation}

In the case of the two interleaved three-level converter legs of the previous section, the SHE-PWM problem can be formulated in two different ways, either as two separate threelevel waveforms (Fig. 4(a)) or as a combined optimisation of a five-level waveform (Fig. 4(b)). In the former case, the fundamental frequency component $\left(\hat{V}_{1 x j}\right)$ of each of the converter legs can be controlled independently to the extend that SHE-PWM solutions can be calculated, although it is typically preferable to share the component equally between the two legs.

A minimisation process for the ZSCC was developed in [18]. However, if a formulation of the circulating current is not explicitly set within the equations, then $i_{c i r c, x}$ and the ZSCC cannot be accurately controlled. As each converter generates a three-level waveform, the SHE-PWM problem is defined as a single system of equations throughout the whole modulation index $(M)$. The restriction on the modulation index is that of $0<M<1$ and the linear modulation index range is defined as $M \in[0, \pi / 4]$ [16]. A sample solution for this particular formulation is shown in Fig. 5. The formulation is based on

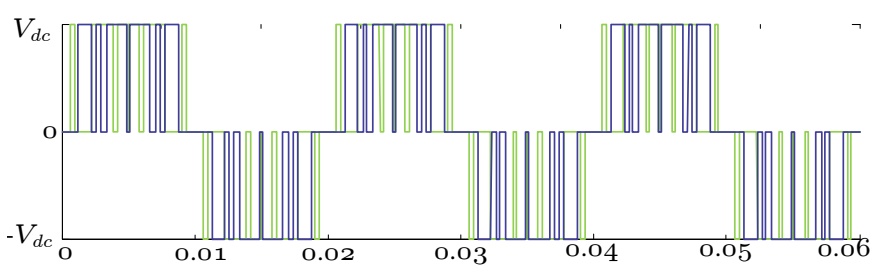

(a)

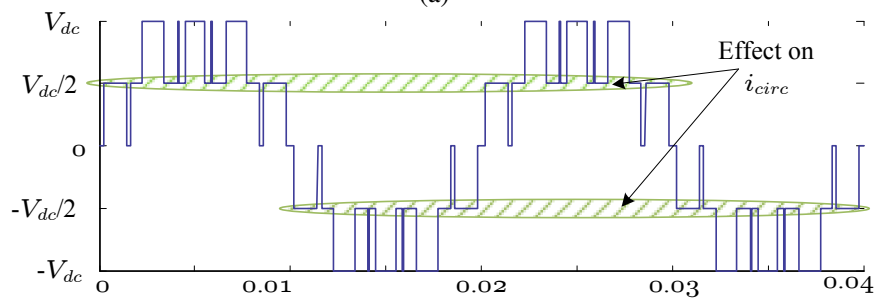

(b)

Fig. 4. Waveforms for SHE-PWM formulation. (a) Two three-level waveforms and (b) combined optimisation based on a five-level waveform.

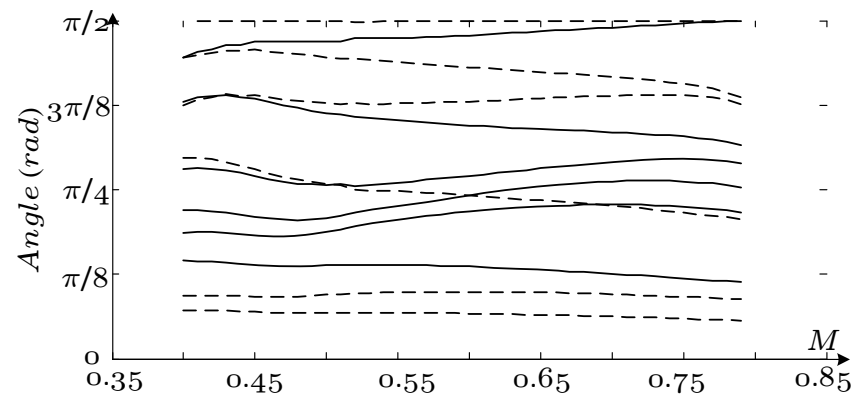

Fig. 5. SHE-PWM solution pattern for three-level waveforms and six transitions per quarter-period, per converter leg (solid line: converter leg 1, dashed line: converter leg 2).

the cancellation of harmonics between the two converter leg voltages, similarly to the single-phase formulation in [20].

An alternative approach to considering each of the threelevel output voltages independently, is to treat the problem as a global optimisation of the output voltage, $v_{x}$. In this case, the five-level formulation needs to consider both the number of transitions within the quarter period $(N)$ as well as their distribution in the different levels of the waveforms [16]. The distribution of the angles to the levels is defined as the number of angles between Levels 0 and $1\left(N_{1}\right)$ and between Levels 1 and $2\left(N_{2}\right)$. As an example, Fig. 4(a) shows the output voltage level in the case of twelve $(N=12)$ switching angles $\left(\alpha_{i}\right)$ per quarter period and a $N_{1} / N_{2}=5 / 7$ distribution between the two levels of the waveform. The system of equations that describe the fundamental frequency $\left(b_{1}\right)$ and higher order $\left(b_{n}\right)$ harmonic components is then defined as:

$$
\begin{gathered}
\sum_{i=1}^{N_{1}}(-1)^{i-1} \cos \left(\alpha_{i}\right)+\sum_{i=N_{1}+1}^{N}(-1)^{i-1} \cos \left(\alpha_{i}\right)=b_{1}, \\
\sum_{i=1}^{N_{1}}(-1)^{i-1} \cos \left(n \alpha_{i}\right)+\sum_{i=N_{1}+1}^{N}(-1)^{i-1} \cos \left(n \alpha_{i}\right)=b_{n},
\end{gathered}
$$

where $i$ is the order of each switching transition, $n \in\{1,5,7, \ldots, 3 N-1\}$ is the harmonic order, $M$ 
is the modulation index, and $N_{1}$ the number of transitions between the zero and the first level of the waveform, typically considered an odd number. In both three-level and fivelevel waveforms, the fundamental frequency component of the output voltage is defined as:

$$
\hat{V}_{1}=\frac{4 M}{\pi} V_{d c}
$$

with $0<M<2$ in the case of the five-level waveform and the linear modulation index range is $M \in[0,2 \pi / 4]$ for the fivelevel waveform. The solutions can be calculated through an iterative optimisation of the cost function which is formulated as:

$$
F\left(\alpha_{1}, \ldots, \alpha_{i}\right)=\left(b_{1}-M\right)^{2}+b_{5}^{2}+b_{7}^{2}+\ldots+b_{3 N-1}^{2},
$$

for even values of $N$ and assuming a cost of zero for the SHEPWM solutions. An additional restriction in the optimisation considers the switching angle placement so that

$$
0<\alpha_{1}<\alpha_{2}<\ldots<\alpha_{N}<\frac{\pi}{2} .
$$

Fig. 6 shows SHE-PWM solution patterns for five-level waveforms with twelve angles per quarter-wave $(\mathrm{QW})$ and different distributions of transitions among the different levels and for different values of $M$. A complete set of five-level solutions for different angles and distributions can be found in [22].

\section{B. Comparison and Evaluation of Solutions}

As described in Section II-B, $i_{c i r c, x}$ is only affected during the $+V_{d c}$ and $-V_{d c}$ voltage levels of the five level waveform (Fig. $4 \mathrm{~b}$ ). The maximum deviation $\left(\Delta i_{c i r c, x}\right)$ of the circulating current during a QW (and due to the imposed QW symmetry for the whole fundamental period) will be observed when state +1 (or symmetrically -1 during the negative half-cycle) is imposed for the longest time interval. This angle interval $\Delta \alpha_{\max }$ is given by:

$$
\Delta \alpha_{\max }=\alpha_{2 g}-\alpha_{2 g-1},
$$

where $g \in\{1, N / 2\}$ and can be evaluated during the calculation of the solutions. Under proper control of the circulating current within each phase, the peak value of $i_{\text {circ }, x}$ will be equal to its maximum deviation which is linked to the solution pattern as:

$$
\hat{i}_{c i r c, x}=\Delta i_{c i r c, x}=\frac{V_{d c}}{2 L} \Delta \alpha_{\max } .
$$

In order to minimise the peak of the circulating current, it is preferable to select SHE-PWM solutions that minimise the time interval at either $+V_{d c} / 2$ or $-V_{d c} / 2$. As SHE-PWM problems typically exhibit multiple solutions, this additional evaluation should be performed between solutions of the same or different distributions for a given $M$ or range of $M$. Referring back to Fig. 6, we can observe the angles that cause the highest deviation and, subsequently, peak $i_{c i r c, x}$ ripple for a given modulation index (highlighted areas of Fig. 6). For example, considering Set 1 of the 7/5 distribution and Set 2 of the $5 / 7$ distribution, the former generates approximately $25 \%$ higher peak ripple current. Similarly, SHE-PWM solutions for

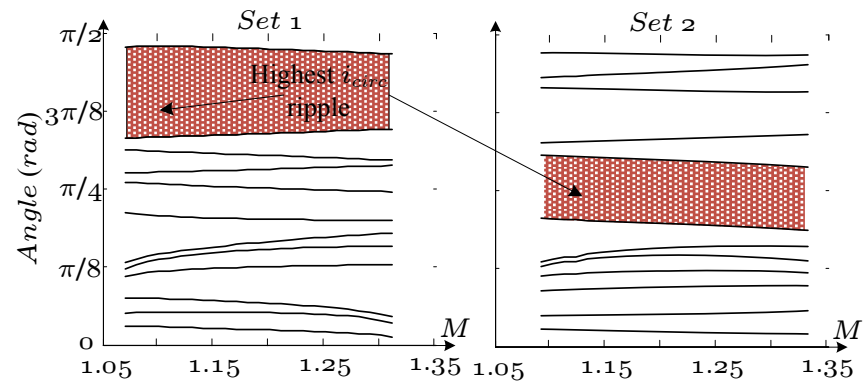

(a)

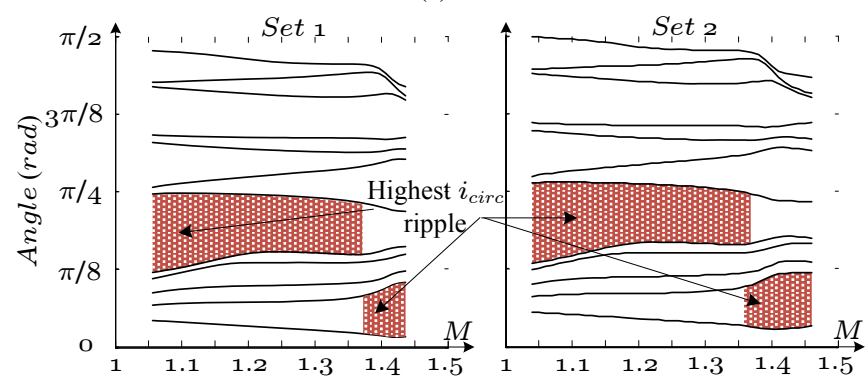

(b)

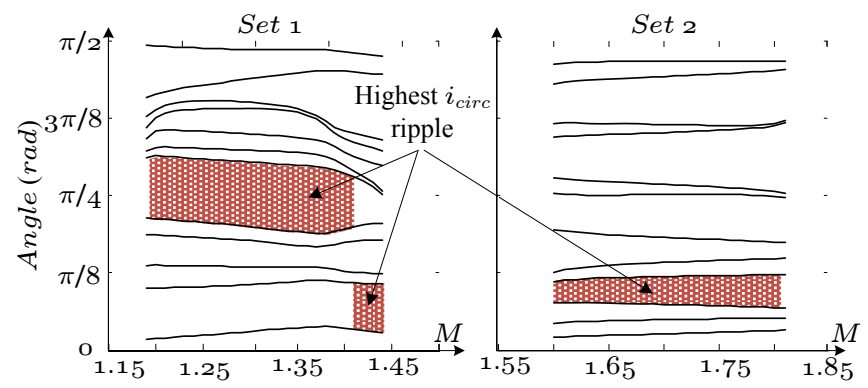

(c)

Fig. 6. SHE-PWM solution patterns and locations of higher $i_{c i r c}$ ripple for a five-level optimised waveform with (a) 7/5 distribution, (b) 5/7 distribution, and (c) 3/9 distribution.

3/9 distribution in two different ranges of $M$ are illustrated in Fig. 6(c). It should also be noted that Set 2 of Fig. 6(c) provides solutions in the overmodulation region which are typically not available when triplen harmonics are controlled.

If a similar approach is assumed for the ZSCC, then the maximum deviation of the circulating current can be found considering the transitions at all three phases and the maximum interval that may contribute to the deviation of the ZSCC. As it will be shown in the simulation results, such a control can minimise the ZSCC but can lead to excessive circulating currents within each phase.

\section{Minimisation of the Circulating Current}

This section presents the configuration of the $i_{c i r c, x}$ controller based on the definitions and restrictions introduced in Section III. The diagram of the proposed controller is shown in Fig. 7 and is split into two sections, the SHE-PWM pattern generation and the selection of the switching states for the control of $i_{c i r c, x}$

The first part decomposes the reference signal $v_{x m}$ into two components: the modulation index $M$ based on the definition of (9) and the phase angle $\theta$. These two values could also 


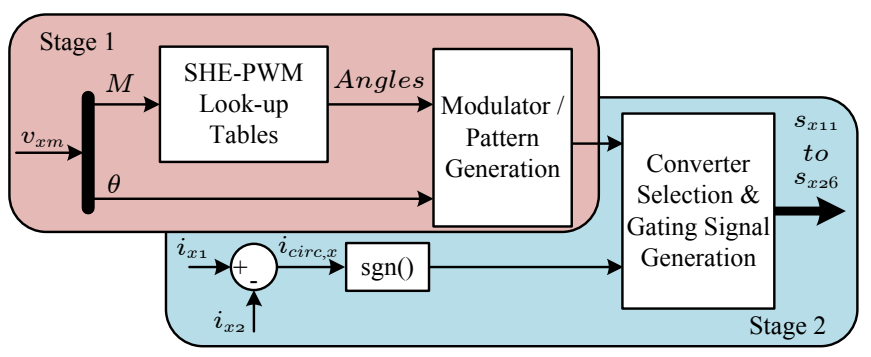

Fig. 7. Implementation of the proposed two-stage circulating current controller for two interleaved converter legs.

be readily available based on way external controllers may be implemented. Based on $M$ and the associated switching angles $\alpha_{i}$, the requested switching pattern is generated and made available to the second stage of the controller. The switching pattern is a normalised switching waveform similar to that of Fig. 4, and provides information related to the required output voltage level of the converter.

The second stage defines the switching states of the two converter legs based on the sign of $i_{\text {circ }}$ and makes use of the relation between switching states and circulating current that was demonstrated in Fig. 3. In order to achieve that, the controller selects the appropriate state when the voltage waveform level is at \pm 1 so that the circulating current is driven towards zero. For example, when the circulating current is positive $\left(i_{x 1}-i_{x 2}>0\right)$ and there is a transition in the required voltage waveform from Stage 1 towards a redundant voltage level, the controller would select the state with -1 effect on the circulating current $\left(\left[0,+V_{d c}\right]\right.$ when at level 1 and $\left[-V_{d c}, 0\right]$ when at level -1$)$. The opposite selection takes place when $i_{\text {circ }}<0$. Because the selection only occurs during transitions in voltage levels, the method does not affect the switching frequency of the converter. The controller regulates the circulating current within one phase only, therefore, three independent controllers are required for a three-phase system.

In a similar way, a minimisation of the ZSCC can be performed considering every switching interval of the three phases and selecting the switching states in such a manner that the current of (5) is driven towards zero. The implementation of the controller for the ZSCC is similar to that of Fig. 7 considering ZSCC instead of $i_{c i r c, x}$.

\section{Simulation Results}

A set of simulation studies are presented to illustrate the three-phase behavior of the proposed controller (Fig. 7) in terms of circulating current and ZSCC. Results on the perphase performance are given in Section VI based on the experimental prototype. The parameters of the three-phase system under study are given in Table I.

A common operating point $(M=1.35$ with common load parameters) is considered under the five-level SHE-PWM of Section IIIA for two different angle distributions (5/7 Set 2 and $3 / 9$ Set 1 ). The circulating current controller of Section IV is also included in the simulation. The load current is common in both cases and is shown in Fig. 8. The main purpose of
TABLE I

PARAmeters of THE Simulated AND ExPERIMENTAl SetuP

\begin{tabular}{c|c|c}
\hline Parameters & Simulation & Experiment \\
\hline dc-link Voltage, $V_{d c}$ & $3 \mathrm{kV}$ & $100 \mathrm{~V}$ \\
\hline dc-link Capacitors, $C_{d c}$ & $3.6 \mathrm{mF}$ & $4.9 \mathrm{mF}$ \\
\hline Leg Inductors (single), $L$ & $5 \mathrm{mH}$ & $1 \mathrm{mH}$ \\
\hline Fundamental frequency, $f_{0}$ & $50 \mathrm{~Hz}$ & $50 \mathrm{~Hz}$ \\
\hline Switching Angles per QW, $N$ & 12 & 12 \\
\hline Load Resistance $R_{\text {load }}$ & $1 \Omega$ & $23.5 \Omega$ \\
\hline Load Inductance $L_{\text {load }}$ & $2 \mathrm{mH}$ & 0 \\
\hline \hline
\end{tabular}

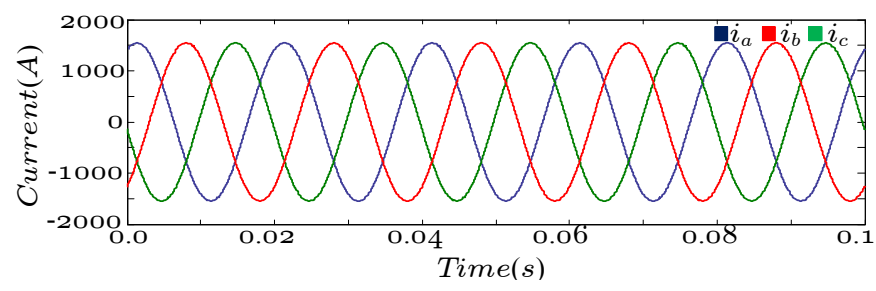

Fig. 8. Three-phase load currents for $M=1.35$.

the results is to demonstrate the effect that the selection of a particular set of solutions can have on the circulating current of each phase and the ZSCC, with the $5 / 7$ distribution exhibiting a larger $\Delta \alpha_{\max }$.

Considering the $5 / 7$ distribution, the large $\Delta \alpha_{\max }$ leads to a higher circulating current deviation (Fig. 9(a)) compared to the 3/9 distribution of Fig. 9(b). The current sharing between the two interleaved legs of the same phase $\left(i_{a 1}\right.$ and $\left.i_{a 2}\right)$ are also significantly different as it was demonstrated in [9]. The ZSCC (also shown in Fig. 9) is also different from that of the 5/7 distribution exhibiting a higher peak value and a characteristic third harmonic. The comparison between the phase circulating currents and the ZSCC indicates that the ZSCC is not the only critical component in the selection of SHE-PWM solution sets. Due to the low switching frequency, the phase circulating currents should be generally considered as they provide a more definite metric for the stresses across the differential mode inductors in interleaved converters.

The proposed controller does not modify any of the switching angles in order not to affect the quality of the waveform, although such modifications may be included in order to facilitate balancing of neutral-point or flying capacitor voltages. The regulation of the neutral-point can also be achieved through $R L C$ balance boosters [23] or active methods. Fig. 10 shows the neutral-point $(N P)$ voltage deviation for the three-phase system for the two cases. The deviation in both cases is similar, yet some slight offset is observed in the case of the $5 / 7$ distribution.

To further illustrate the difference between controlling the $i_{\text {circ }, x}$ and ZSCC, the previous simulation is repeated using the ZSCC of (5) to determine the switching state of all three phases of the converter. This approach reduces the peak value of the ZSCC (Fig. 11) compared to the per-phase control. However, as there is no explicit control over the phase circulating currents $i_{c i r c, x}$, their value may reach unacceptably 

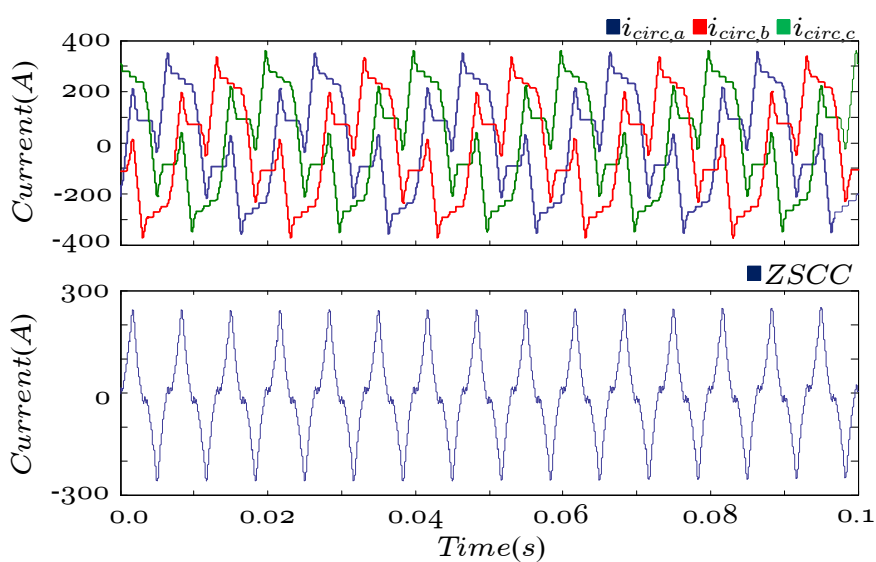

(a)
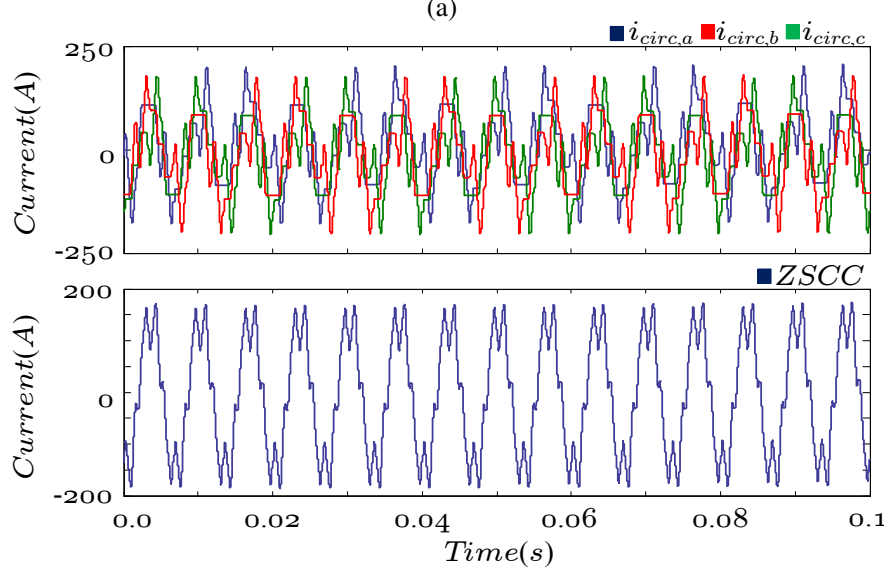

(b)

Fig. 9. Circulating current and ZSCC for $M=1.35$. (a) 5/7 distribution Set 2, (b) 3/9 distribution Set 1

large values, well above the output current of each phase of the converter.

\section{EXPERIMENTAL RESULTS}

In order to experimentally validate the operation of the interleaved converter legs under SHE-PWM and the proposed circulating current controller, a single-phase setup consisting of two three-level ANPC converter legs (Fig. 12(a)) has been built in our laboratories using Semikron SKM145GB176D 1700V IGBT modules. The prototype, shown in Fig. 12(b), is rated at 10kVA with a nominal dc-link voltage of up to $1300 \mathrm{~V}$ and a maximum switching frequency of $12 \mathrm{kHz}$, although these limits are not reached for the purpose of this experiment. The common dc-link of the interleaved converter legs is supplied by a Sorensen DLM300-13M dc power supply, while the control and protection functions of the converter and the interleaved SHE-PWM are implemented in a dSPACE 1006 platform. The parameters of the converter and the load are given in Table I. It should be noted that the value of the inductors $L$ used in the experiment is selected in such a way that the circulating current $i_{\text {circ }}$ has a more prominent effect than normal.

The effect of the SHE-PWM pattern in the circulating current amongst interleaved converters and the importance of selecting an appropriate pattern that reduces its peak is

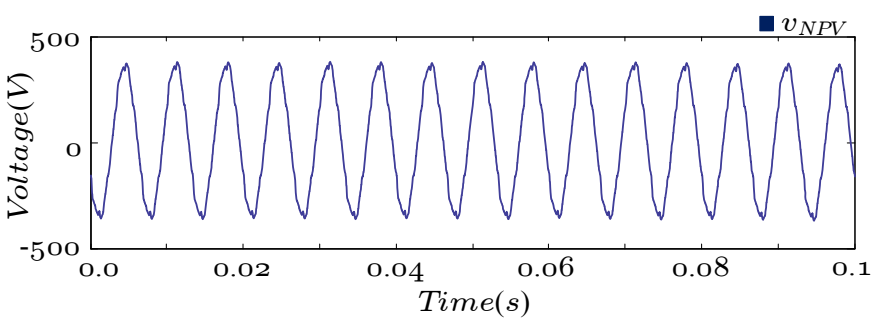

(a)

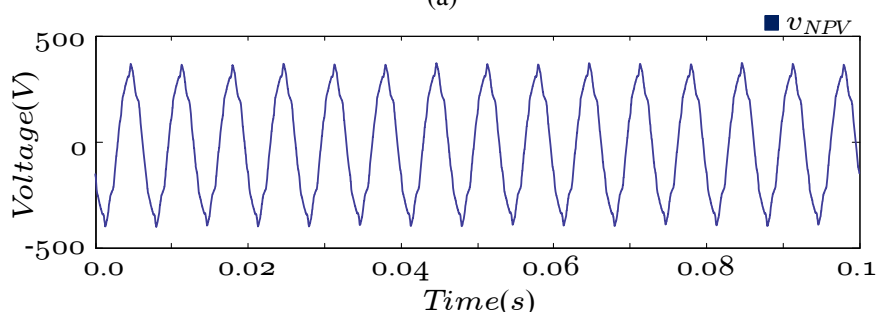

(b)

Fig. 10. Neutral-point voltage deviation for $M=1.35$. (a) $5 / 7$ distribution Set 2, (b) 3/9 distribution Set 1 .

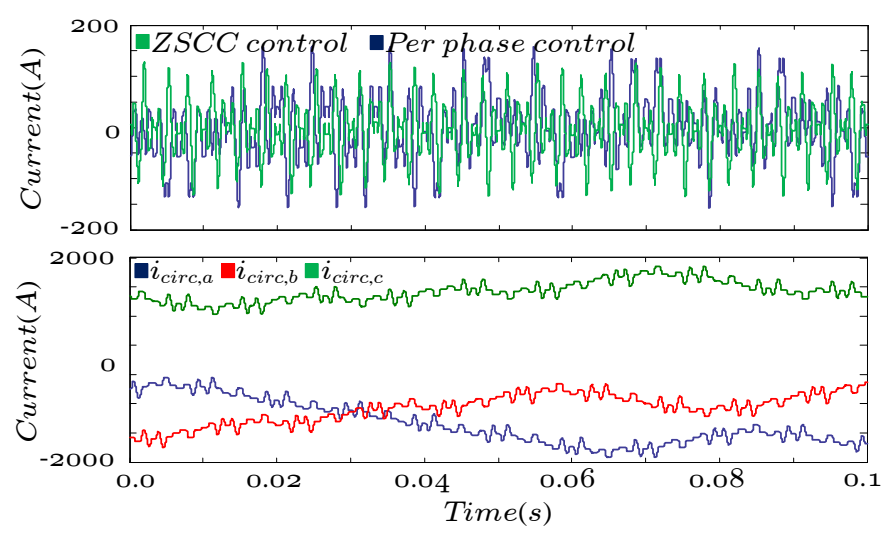

Fig. 11. Phase and zero sequence circulating currents under control of the ZSCC.

illustrated by the results in Figs. 13-16. Assuming a common modulation index $M=1.35$, Figs. 13-16 show the load and leg voltages as well as the load, leg and circulating currents for different SHE-PWM patterns. The common modulation index used in the experiment means that the amplitude of the fundamental component of the load voltage, $v_{a}$ and current $i_{a}$ is common across all cases.

With $N=12$ angles per $\mathrm{QW}$, the first non-triplen harmonic in the output voltage spectrum is the $37 \mathrm{th}(1850 \mathrm{~Hz})$. This harmonic is marked in the presented FFTs of all experimental results. A particularly interesting example is that of Fig. 13, for a $7 / 5$ distribution, where the third harmonic is almost negligible and the predominant triplen harmonic is the 9th $(450 \mathrm{~Hz})$. The amplitude of the non-eliminated harmonics is defined by the set of solutions and is not related to the control or elimination of the circulating current between the two converter legs.

Comparing the circulating currents in Figs. 13(b) to 16(b), it can be observed that Set 2 of 5/7 distribution (Fig. 15) results in the highest circulating current between the converter legs, mainly due to the large time interval at the $+V_{d c} / 2$ 


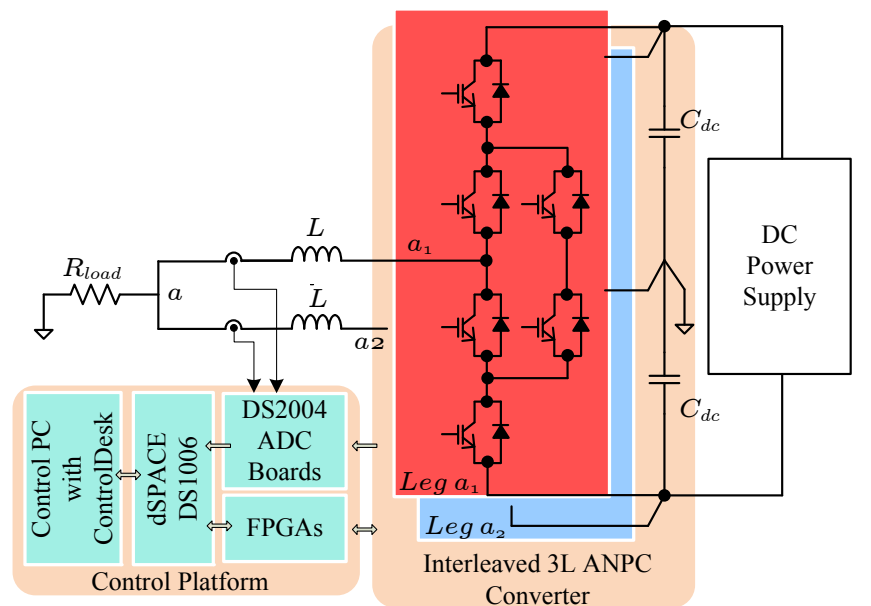

(a)

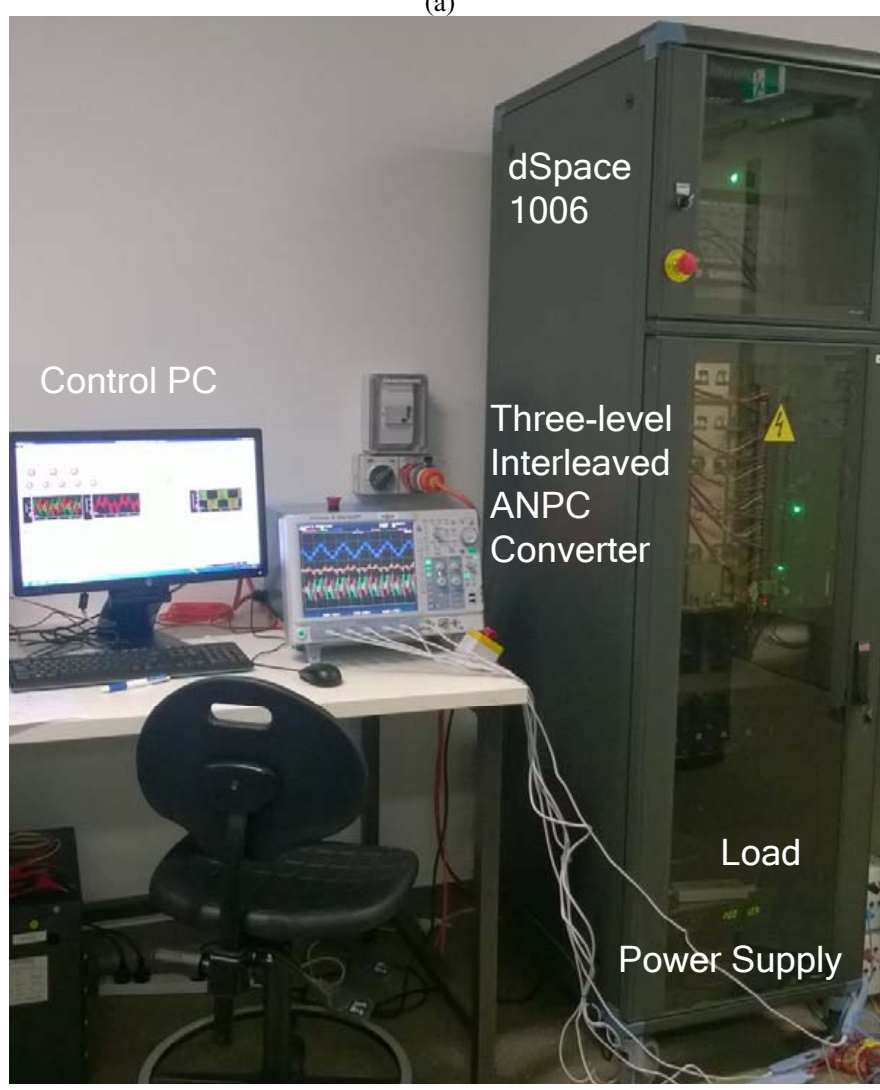

(b)

Fig. 12. Experimental setup, (a) Schematic diagram and, (b) Photo.

and $-V_{d c} / 2$ voltage level. In order to control the circulating current, neither the transitions or the power can be shared equally between the two converter legs, as shown in Fig. 15(a). Yet, the harmonic spectrum of the five-level load voltage, $v_{a}$, shows relatively low triplen harmonics.

As the formulation of the problem does not consider elimination of triplen harmonics, full utilisation of the dclink voltage can be achieved and solutions well within the overmodulation $(M>2 \pi / 4)$ range can be calculated [16]. Fig. 17(a) shows the output phase voltage, $v_{a}$ and its FFT as well as the converter leg voltages for an angle distribution of $3 / 9$ at $M=1.75$. Given the selected solution pattern (Set 2

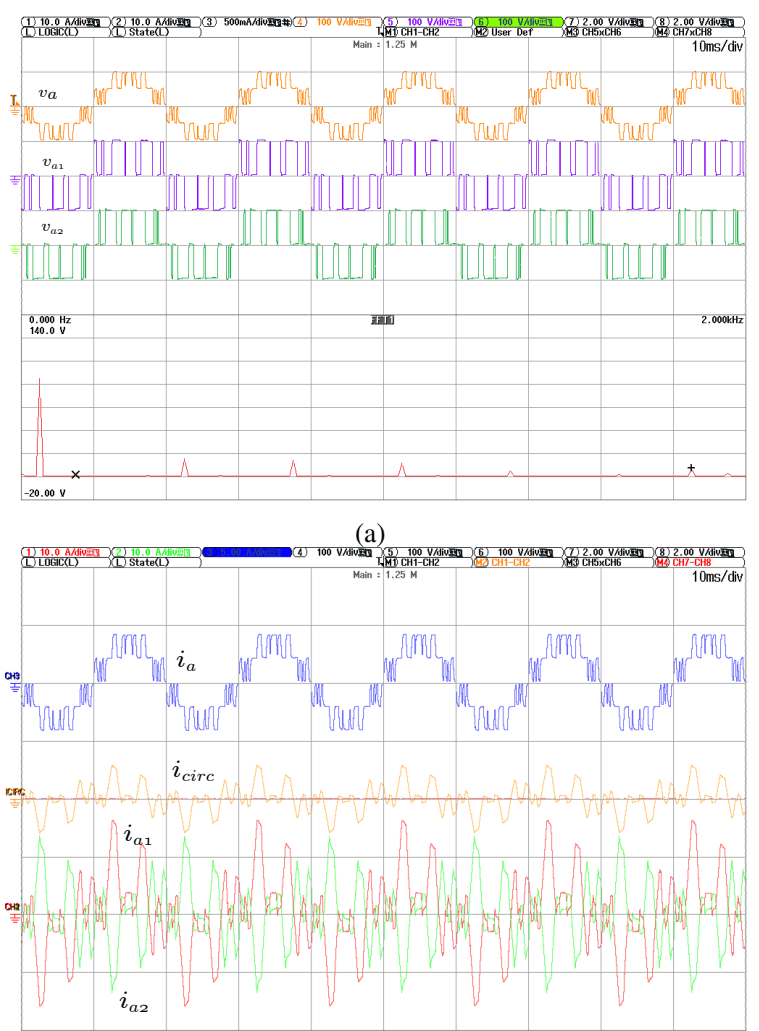

(b)

Fig. 13. Experimental waveforms for $7 / 5$ distribution and $M=1.35$. (a) Load and leg voltages and load voltage harmonic spectrum, and (b) Load, leg and circulating currents. $\mathrm{CH} 1, \mathrm{CH} 2$ : 10A/div, $\mathrm{CH} 3$ : 5A/div, $\mathrm{CH} 4, \mathrm{CH} 5, \mathrm{CH} 6$ : $100 \mathrm{~V} / \mathrm{div}$.

of Fig. 6(c)) and the distribution of angles within the quarterperiod, the converter currents of Fig. 17(b) $\left(i_{a 1}\right.$ and $\left.i_{a 2}\right)$ are well matched and $i_{c i r c, a}$ remains well controlled.

The presented simulation and experimental results illustrate a number of aspects when applying SHE-PWM in interleaved converter legs. Firstly, the amplitude of the harmonics within the circulating currents is defined by the SHE-PWM solution sets and certain sets of solutions may have very low amplitudes of certain triplen harmonics. As with any application of SHEPWM, an evaluation of the available sets of solutions for a particular application is required prior to actual implementation. Secondly, reduction of the circulating current within the phases does not imply minimisation of the ZSCC. Finally, as the proposed controller selects the states of the converter in order to minimise the circulating current within each phase, and in contrast with previous methods, the circulating current is no longer a periodical waveform.

\section{CONCLUSION}

Interleaved parallel connection of converter legs provides an efficient way of increasing the maximum power through each converter without parallel connection of semiconductors. In order to fully utilise the harmonic characteristics of the interleaved configuration, different voltages are generated between the converter legs. Such an operation yields a multilevel waveform but also results in circulating currents within the converter legs as well as a ZSCC, all of which should be 

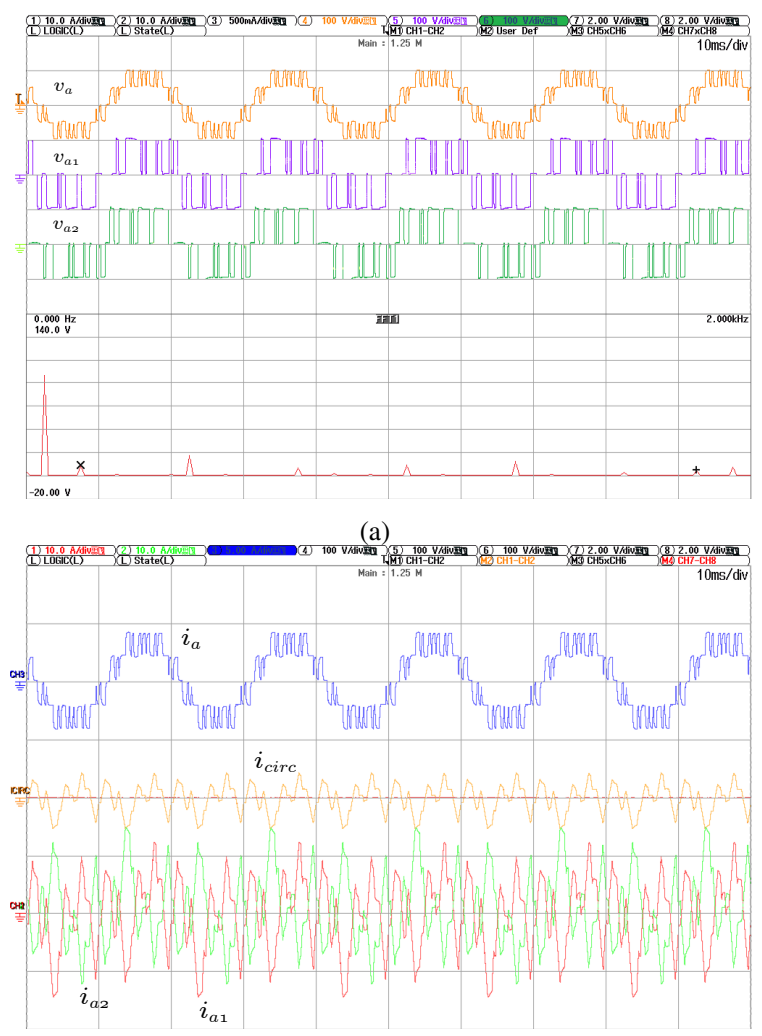

(b)

Fig. 14. Experimental waveforms for $5 / 7$ distribution and $M=1.35$, Set 1 (a) Load and leg voltages and load voltage harmonic spectrum, and (b) Load leg and circulating currents. $\mathrm{CH} 1, \mathrm{CH} 2$ : 10A/div, $\mathrm{CH} 3$ : 5A/div, $\mathrm{CH} 4, \mathrm{CH}$, CH6: 100V/div.

minimised or kept well-regulated for efficient operation of the system. These tasks become more challenging at low switching frequencies as unequal voltages between the interleaved converter legs are generally applied for longer time intervals.

A controller to reduce the circulating current in each phase of two interleaved three-level neutral-point clamped converter legs under SHE-PWM, has been proposed in this paper. By selecting the switching states of the legs based on the phase circulating current $i_{\text {circ, } x}$ it is possible to limit the currents through each converter leg to a minimum and, at the same time, regulate the ZSCC. The proposed controller is further complemented by an evaluation and selection of SHE-PWM patterns that generate low ripple in the circulating currents. Simulation and extended experimental results from a singlephase laboratory prototype converter are provided to confirm the performance of SHE-PWM and the proposed current controller.

\section{REFERENCES}

[1] T. Kawabata and S. Higashino, "Parallel operation of voltage source inverters," IEEE Trans. Appl. Ind., vol. 24, no. 2, pp. 281-287, Mar 1988.

[2] Z. Ye, D. Boroyevich, J.-Y. Choi, and F. Lee, "Control of circulating current in two parallel three-phase boost rectifiers," IEEE Trans. Power Electron., vol. 17, no. 5, pp. 609-615, Sep 2002.

[3] H. Mori, K. Matsui, K. Kondo, I. Yamamoto, and M. Hasegawa, "Parallel-connected five-level pwm inverters," IEEE Trans. Power Electron., vol. 18, no. 1, pp. 173-179, Jan 2003.
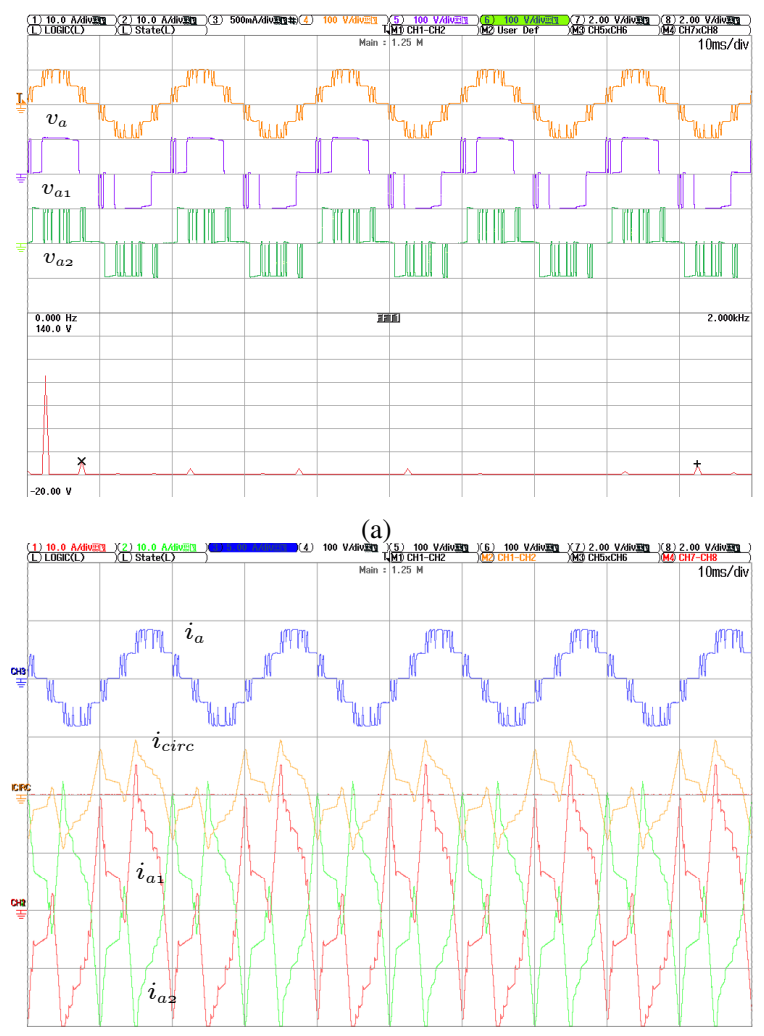

(b)

Fig. 15. Experimental waveforms for $5 / 7$ distribution and $M=1.35$, Set 2 (a) Load and leg voltages and load voltage harmonic spectrum, and (b) Load, leg and circulating currents. $\mathrm{CH} 1, \mathrm{CH} 2$ : 10A/div, $\mathrm{CH} 3$ : 5A/div, $\mathrm{CH} 4, \mathrm{CH}$, CH6: 100 V/div.

[4] B. Ge, X. Lu, X. Yu, M. Zhang, and F. Z. Peng, "Multiphase-leg coupling current balancer for parallel operation of multiple mw power modules," IEEE Trans. Ind. Electron., vol. 61, no. 3, pp. 1147-1157, March 2014.

[5] G. Capella, J. Pou, S. Ceballos, J. Zaragoza, and V. Agelidis, "Currentbalancing technique for interleaved voltage source inverters with magnetically coupled legs connected in parallel," IEEE Trans. Ind. Electron. vol. 62, no. 3, pp. 1335-1344, March 2015.

[6] T. Lazzarin, G. Bauer, and I. Barbi, "A control strategy for parallel operation of single-phase voltage source inverters: Analysis, design and experimental results," IEEE Trans. Ind. Electron., vol. 60, no. 6, pp. 2194-2204, June 2013.

[7] J. Ewanchuk and J. Salmon, "Three-limb coupled inductor operation for paralleled multi-level three-phase voltage sourced inverters," IEEE Trans. Ind. Electron., vol. 60, no. 5, pp. 1979-1988, May 2013.

[8] J. Pou, S. Ceballos, G. Konstantinou, G. J. Capella, and V. G. Agelidis, "Control strategy to balance operation of parallel connected legs of modular multilevel converters," in Industrial Electronics (ISIE), 2013 IEEE International Symposium on, May 2013, pp. 1-7.

[9] G. Konstantinou, J. Pou, G. Capella, S. Ceballos, and V. Agelidis, "Reducing circulating currents in interleaved converter legs under selective harmonic elimination pulse-width modulation," in Industrial Technology (ICIT), 2015 IEEE International Conference on, March 2015, pp. 11361141.

[10] J. Shen, S. Schroder, B. Qu, Y. Zhang, K. Chen, F. Zhang, Y. Li, Y. Liu P. Dai, and R. Zhang, "A high-performance $2 \times 27$ mva machine test bench based on multilevel igct converters," IEEE Trans. Appl. Ind., vol. PP, no. 99, pp. 1-1, 2015.

[11] X. Yu and A. Khambadkone, "Reliability analysis and cost optimization of parallel-inverter system,” IEEE Trans. Ind. Electron., vol. 59, no. 10, pp. 3881-3889, Oct 2012.

[12] T.-P. Chen, "Zero-sequence circulating current reduction method for parallel hepwm inverters between ac bus and dc bus," IEEE Trans. Ind. Electron., vol. 59, no. 1, pp. 290-300, Jan 2012.

[13] G. Capella, J. Pou, S. Ceballos, G. Konstantinou, J. Zaragoza, and V. Agelidis, "Enhanced phase-shifted pwm carrier disposition for inter- 

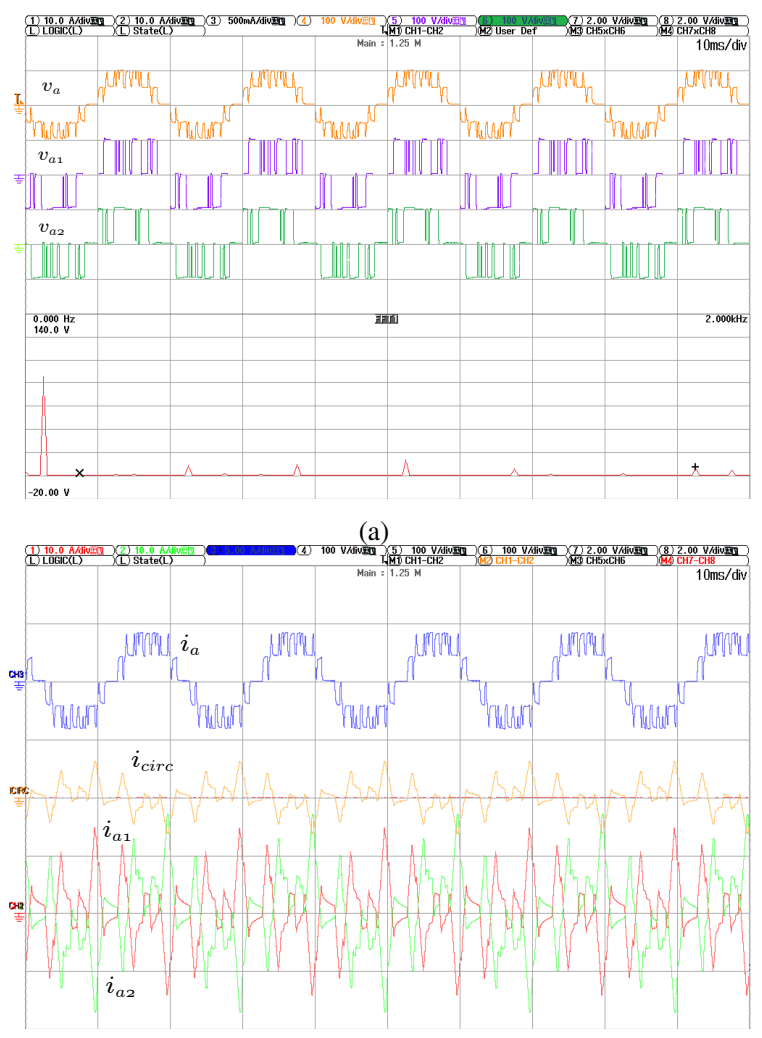

(b)

Fig. 16. Experimental waveforms for $3 / 9$ distribution and $M=1.35$, Set 1 . (a) Load and leg voltages and load voltage harmonic spectrum, and (b) Load, leg and circulating currents. $\mathrm{CH} 1, \mathrm{CH} 2$ : 10A/div, $\mathrm{CH} 3$ : 5A/div, $\mathrm{CH} 4, \mathrm{CH}$, CH6: 100V/div.

leaved voltage-source inverters," IEEE Trans. Power Electron., vol. 30 no. 3, pp. 1121-1125, March 2015.

[14] Y. Zhou and H. Nian, "Zero-sequence current suppression strategy of open-winding pmsg system with common dc bus based on zero vector redistribution," IEEE Trans. Ind. Electron., vol. 62, no. 6, pp. 3399_ 3408, June 2015.

[15] Y.-H. Liao and H. C. Chen, "Simplified pwm with switching constrain method to prevent circulating currents for paralleled bidirectional ac/dc converters in grid-tied system using graphic analysis," IEEE Trans. Ind. Electron., vol. 62, no. 7, pp. 4573-4586, July 2015.

[16] M. Dahidah, G. Konstantinou, and V. Agelidis, "A review of multileve selective harmonic elimination pwm: Formulations, solving algorithms, implementation and applications," IEEE Trans. Power Electron., vol. 30, no. 8, pp. 4091-4106, Aug 2015.

[17] M. Narimani and G. Moschopoulos, "Three-phase multi-module vsis using she-pwm with reduced zero-sequence circulating current," in Energy Conversion Congress and Exposition (ECCE), 2012 IEEE, Sept 2012, pp. 2859-2865.

[18] — , "Three-phase multimodule vsis using she-pwm to reduce zerosequence circulating current," IEEE Trans. Ind. Electron., vol. 61, no. 4, pp. 1659-1668, April 2014.
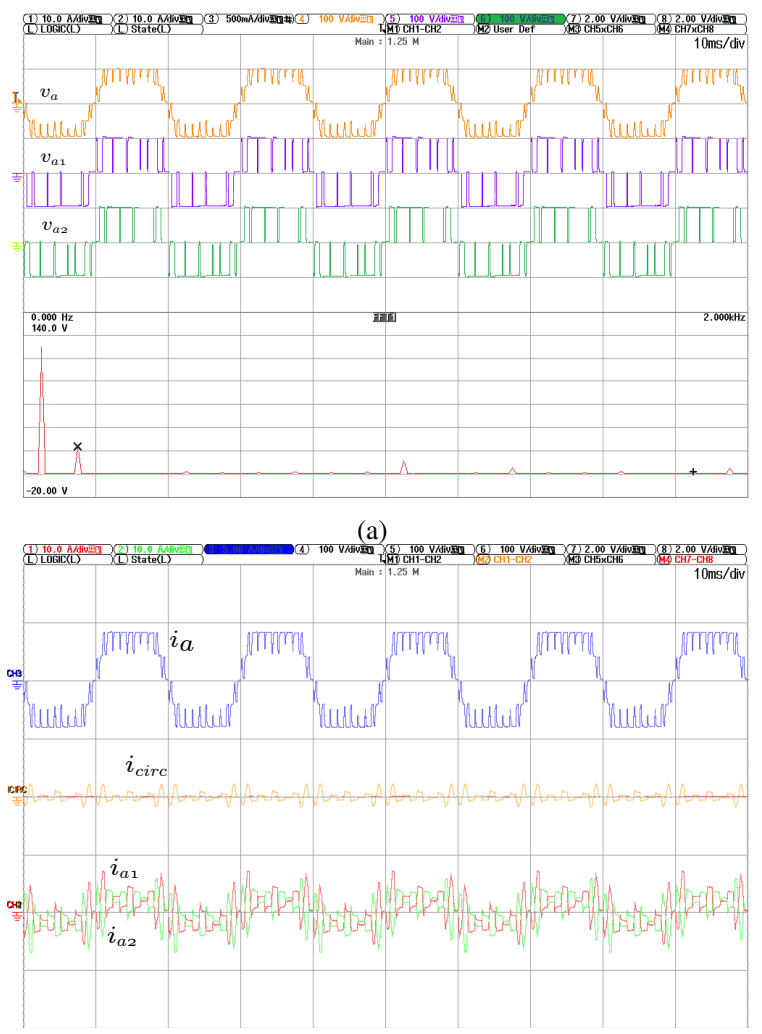

(b)

Fig. 17. Experimental waveforms for $3 / 9$ distribution and $M=1.75$. (a) Load and leg voltages and load voltage harmonic spectrum, (b) Load, leg and circulating currents. $\mathrm{CH} 1, \mathrm{CH} 2$ : $10 \mathrm{~A} / \mathrm{div}, \mathrm{CH} 3$ : $5 \mathrm{~A} / \mathrm{div}, \mathrm{CH} 4, \mathrm{CH} 5, \mathrm{CH} 6$ : $100 \mathrm{~V} / \mathrm{div}$.

[19] — - "Improved method for paralleling reduced switch vsi modules: Harmonic content and circulating current," IEEE Trans. Power Electron., vol. 29, no. 7, pp. 3308-3317, July 2014.

[20] G. Konstantinou, V. Agelidis, and J. Pou, "Theoretical considerations for single-phase interleaved converters operated with she-pwm," IEEE Trans. Power Electron., vol. 29, no. 10, pp. 5124-5128, Oct 2014.

[21] Y. Zhang, Y. Kang, and J. Chen, "The zero-sequence circulating currents between parallel three-phase inverters with three-pole transformers and reactors," in Applied Power Electronics Conference and Exposition, 2006. APEC '06. Twenty-First Annual IEEE, March 2006, pp. 7 pp.-

[22] G. Konstantinou, S. Pulikanti, and V. Agelidis, "Harmonic elimination control of a five-level dc-ac cascaded h-bridge hybrid inverter," in Power Electronics for Distributed Generation Systems (PEDG), 2010 2nd IEEE International Symposium on, June 2010, pp. 352-357.

[23] R. Stala, "A natural dc-link voltage balancing of diode-clamped inverters in parallel systems," IEEE Trans. Ind. Electron., vol. 60, no. 11, pp. 5008-5018, Nov 2013 